\title{
MAMOUDOU SY
}

\section{»Peuplades« ou descendants de chorfas*? Représentations au XIX ${ }^{\mathrm{e}}$ siècle des peuples de la Sénégambie septentrionale}

La Sénégambie est définie comme la région s'étendant grosso modo de la Mauritanie méridionale aux deux Guinées (Conakry et Bissau) du nord au sud. À la Sénégambie septentrionale correspondent actuellement la Mauritanie méridionale et le Sénégal septentrional et central. Nous nous intéressons pour notre part à l'État précolonial du Fuuta Tooro et des régions voisines, comme le Soudan occidental, ayant accueilli les Fuutankoobe $^{1}$ au $\mathrm{XIX}^{\mathrm{e}}$ siècle. Certaines de ces populations, de par leurs récits d'origines, se disent originaires de l'épicentre du monde musulman et déclarent n'être liées généalogiquement à aucun convertisseur arabe ou berbère. Quel est le discours officiel de la représentation de ces peuples africains au XIX $\mathrm{C}^{\mathrm{e}}$ siècle? Comment évolua entre 1820 et 1891 la vision que l'administration coloniale française, les missionnaires et explorateurs eurent des populations de la Sénégambie septentrionale? Telles sont les questions centrales auxquelles nous allons répondre, en faisant appel aux documents d'archives coloniales françaises, aux récits de voyages et à la tradition orale.

\section{L’ÉLITE POLITIQUE ET RELIGIEUSE ET SA REPRÉSENTATION}

La communauté dirigée par des marabouts ${ }^{2}$ descendants d'agriculteurs installée dans un territoire situé dans la vallée du Sénégal, entre les villes actuelles de Dagana (région de Saint-Louis) et de Dembankané (région de Matam), prend le pouvoir en 1776 des mains d'une aristocratie dite des Satigi qui sont des Fulbe comme eux. Certains d'entre eux se considérèrent supérieurs à leurs frères de race noire en se construisant un nouveau récit des origines qu'ils codifièrent et dont ils confièrent la vulgarisation à un

* »Chorfas « signifie les descendants du prophète de l'Islam, Mohamed. Le mot est dérivé de »chérif« ou »sharif«.

1 Habitants du Fuuta Tooro. Ils sont très majoritairement des Toucouleurs. On note une minorité Wolof et Soninke aux extrémités occidentale et orientale.

2 Ce terme viendrait de murabit. Il s'agit d'une sainte personne qui entretient une relation spéciale avec Dieu. Ce qui lui donne le pouvoir de transmettre la grâce de Dieu vers la communauté des croyants. Lire Julia ClanCY-SMith, Rebel and Saint. Muslim Notables, Populist Protest, Colonial Encounters (Algeria and Tunisia, 1800-1904), Berkeley, Los Angeles, Oxford 1997. 
groupe social des gawlo ou gewel (griots). Les nouveaux gouvernants du Fuuta Tooro se trouvèrent de nouveaux ascendants provenant d'Asie ${ }^{3}$.

Prenons l'exemple de quelques-unes des familles. Les Kan font remonter leurs origines les plus lointaines jusqu'à un certain Aayel, qui aurait quitté Damas pour s'installer au Macina (république du Mali), puis à Rachid (république de Mauritanie), Tulde Gasama (Tendagassame), enfin il se fixa à Njibeniyidi où il y avait sept mosquées. Les sources orales restent imprécises sur l'identité de ses ascendants et les dates exactes d'implantation des migrants.

Les Lih auraient pour ancêtre Qadim Ely ${ }^{4}$. Les Sih auraient pour ascendant un certain Shems Eddin. T.P. Mbow, plus explicite, nous donne une version qui correspond à la perception réelle que ces populations ont de leurs récits d'origine:

Les deux branches de Ly sont apparentées aux Blancs. Leur ancêtre fut Diam Diam Fadala [...]. Thierno Wanewanebe et Thierno Sadel descendent de Weynde Dieng [...]. Ils sont originaires de l'Égypte. Les Kane sont originaires de Dimask Sam /Damas. Thierno Tillere, Elimane Belinabé, Tapsirou Hamat N'diaye Hane et Amar Bella Racine sont des descendants de Mouhamadou Bousmiyou, un compagnon du prophète Mohamed. Ardo Ururbe, Ceerno Siwol-Nabadji et Ardo Bantou sont des descendants de Ougbatou Boun Amri, un compagnon du Prophète. Thierno Ngapougou, Barobé Diakel et les Silla sont des anciens Sarakollés et disent que leur ancêtre fut Hamdiatou Boun Abduul Motalib, un autre compagnon du Prophète. Elimane Rindiaw est originaire de Médine, ses ancêtres sont de Bagdad. Thierno Siwol Agnam est originaire d'un lieu nommé Sowoïla, un marigot qui se trouve prés de Médine en Arabie. Son ancêtre fut le courageux Oumar Ibn Khattab, un compagnon du Prophète 5 .

Nous sommes en présence d'une compétition pour s'attribuer les origines les plus proches du foyer originel du monde musulman ${ }^{6}$. Un historien sénégalais, Cheikh Moussa Kamara, s'est interrogé à juste titre sur cette revendication d'altérité: »Ce qui est étrange, c'est que si les gens du Massina et ceux du Fouta Jaloo sont fiers d'être peuls, ceux du Fouta Tooro, en revanche, ne le sont pas. Se considérant comme supé-

$3 »$ La saisie de l'origine des Toucouleurs est une des questions centrales de toute recherche sur le Takrur ou le Fouta Toro. C'est une question difficile, compliquée à la fois par l'état actuel des enquêtes et la forte dose d'investissement idéologique qu'elle occasionne«. Abdourahmane BA, Le Takrur, des origines à la conquête par le Mali (VI $-\mathrm{XIII}^{\mathrm{e}}$ siècles), Dakar 2002, p. 60.

4 À l'image des grandes familles toorobbe, les Lih déroulent un long arbre généalogique qui les rattache à de célèbres tribus arabes. Comme chez les Kan avec Hamet Juuldo Kan, c'est à partir de Jam Lih que se fit la dispersion de ses enfants à travers la Sénégambie: Juma Jam Lih s'installa à Salde Tebegut, Mbaran Jam Lih à Fanay et Ciloň, Al Hassan Jam Lih à Salde Tebegut, Ibrahim Jam Lih à Donnay, Yakuba Jam Lih à Mboyo, Ali Jam Lih à Pete, Mamadu Jam Lih à Jaaba, Mboyeydu Jam Lih à Dara Halaybe, Omar Jam Lih et Yusuf Jam Lih à Cilon̆, Molle Jam Lih à Jaranguel, Fatimata Jam Lih à Halwar et enfin Ifra Jam Lih devint pullo. Voir interviews Dieynaba Ciré Kane et Coumba Dada Kane à Jaaňum, novembre 2001 et novembre 2006.

5 Thierno Pathé dit Abdourahmane Mbow, interviewé par David Robinson en 1968. Cassette consultée aux Archives of Traditional Music, Indiana University, juillet 2001.

6 Voir Jean Devisse, Islam et ethnies en Afrique, dans: Jean-Pierre ChrÉTIEN, Georges PRUNIER (dir.), Les ethnies ont une histoire, Paris 1989, p. 103-115, ici p. 114-115. 
rieurs aux Peuls, ils ne veulent pas qu'on les identifie avec ceux-ci. Quelle chose étrange «?

\section{LA MAÎTRISE DU CORAN COMME ÉLÉMENT CENTRAL DE L'ALTÉRITÉ LE JANGGI LAAMI}

Le janggi laami ${ }^{8}$ est une stratégie de légitimation et de conservation du savoir et du pouvoir. Le nouveau groupe qui a pris le pouvoir au Fuuta Tooro à la fin du XVIII siècle partage en commun la fréquentation des écoles coraniques appelées dudal ou daara. Le groupe se renforce par l'arrivée dans la moyenne vallée du Sénégal de populations non pulaarophones, qui vont, par rapport au contexte politique, changer de statut: c'est le cas des Wan, des Kebbe, des Jeng, des Saaxo, des Baro, etc.

Ainsi l'appellation tooroodo sert à la fois de référent linguistique et religieux, car il indique d'abord une mutation de l'état de non-locuteur de la langue pulaar à celui de néo-pulaarophone, ensuite de l'état de >païen ‘ à celui de musulman et enfin de celui de musulman non initié aux sciences coraniques à celui d'érudit. En effet, le berger peul devenu fidèle observateur des règles de l'islam se sédentarise en général. Il est alors nommé par le terme tooroodo, qui est dès lors marqué par une ambiguïté. Au sens large, tooroodo désigne un pullo qui s'est sédentarisé. Il peut être gawlo (griot), cubbalo (pêcheur), maabo (tisserand) etc. Pris dans ce sens, le terme tooroodo se différencie du terme pullo, qui renvoie à l'éleveur nomade.

Étymologiquement, tooroodo provient du verbe toraade, c'est-à-dire »mendier«. Les pulaarophones de la moyenne vallée du Sénégal utilisent eux le verbe yelaade. Les jeunes étudiants en sciences coraniques (taalibabe ou talibe) devaient mendier leur nourriture devant chaque concession avec une sébile ou une petite calebasse devant contenir des restes de nourritures, des céréales etc. Il s'oppose au terme ceddo qui désigne en pulaar un non-pulaarophone.

L'existence d'un État bien organisé dans le Fuuta Tooro à partir de 1776, à la tête duquel se trouvaient les toorobbe ${ }^{9}$ ainsi que la compétition pour l'accès à la terre (surtout les kolaade du Waalo), seraient les éléments explicatifs de la confection de nouveaux récits sur les origines au $\mathrm{XIX}^{\mathrm{e}}$ siècle. Ainsi chaque grande famille du Fuuta Tooro voudrait se rattacher à celle du prophète de l'islam. Il y a aussi la concurrence ıgénéalogiqueく vis-à-vis des familles chérifiennes blanches de la rive droite du fleuve Sénégal: les Berbères et les Arabes.

7 Jean Schmitz (dir.), Florilège au jardin de l'histoire des Noirs. Zuhûr al Basatin, tome 1: L'Aristocratie peule et la révolution des clercs musulmans (vallée du Sénégal), Paris 1998, p. 425.

8 Il s'agit d'un mot composé du pulaar parlé dans la vallée du Sénégal. Il signifie littéralement gouverner grâce à ses capacités intellectuelles et à la maîtrise des sciences islamiques.

9 C'est le pluriel de tooroodo, un membre du groupe social des toorobbe. 


\section{DE LA PERCEPTION DE L'ALTÉRITÉ SOCIALE, GÉOGRAPHIQUE ET RELIGIEUSE}

Les écrits relatifs aux origines et à la classification des Fulbe sont marqués jusqu'ici par deux insuffisances. La première est la différenciation faite entre les Fulbe dits »rouges et pastoraux, nomades et païens « 10 et les Fulbe »noirs, sédentaires et islamisés, bâtisseurs d'États «11. La seconde est que les travaux qui leur sont consacrés sont marqués par la concentration des recherches sur un seul groupe sans explorer les connections.

Ce critère classificatoire faisant référence à l'appartenance ou non à l'Islam remonterait au $\mathrm{X}^{\mathrm{e}}$ siècle ${ }^{12}$. Il est hérité des auteurs arabes tels que $\mathrm{Al}$ Bakri ${ }^{13}$, Ahmed Baba et Al-Maghili14 ${ }^{14}$ Le terme »Tukulër«, forme francisée de l'arabe »Tekrur« ou »Takruri«, fut utilisé par les voyageurs européens qui transformèrent le toponyme en un groupe sethniquer spécifique ${ }^{15}$. Ca Da Mosto, qui connaissait les Sereer, les Fulbe, les Wangara et les Wolof, fut le premier à désigner ce peuple par le terme »Thucaror «16 au $\mathrm{XV}^{\mathrm{e}}$ siècle. Il s'agit aussi d'une déformation de »Takrour« ou »Takrouri«. Cependant, ce terme n'a jamais désigné un groupe ethnique spécifique.

Ainsi dès 1339, une carte de l'Afrique et de l'archipel canarien établie par Dulcert porte la mention de »Tochoror «. En 1375, sur l'Atlas catalan figure une vignette avec le toponyme »Tacorom« ou »Takrur«17. En 1506, Don Pacheco établit une carte où figure »le royaume de Tucurol«. Le passage de la forme arabe à la forme européenne s'est accompagné d'un glissement sémantique. Il sert à partir de ce moment à désigner un peuple de la vallée du Sénégal, aux XVII ${ }^{\mathrm{e}}$ et XVIII ${ }^{\mathrm{e}}$ siècles notamment. Dans la première moitié du $\mathrm{XIX}^{\mathrm{e}}$ siècle, la confusion persistera, lorsqu'en Sénégambie septen-

10 Derrick J. Stenning, Cattle Values and Islamic Values in a Pastoral Population, dans: Ioan M. LEWIS (dir.), Islam in Tropical Africa, Oxford 1966, p. 194-205.

11 Jean SCHMitz, Histoire savante et formes spatio-généalogiques de la mémoire (Haalpulaar de la vallée du Sénégal), dans: Cahier des sciences humaines 26 (1990), p. 490.

12 »C'est un fait généralement admis: jusqu'au $\mathrm{XI}^{\mathrm{e}}$ siècle - peut-être parfois seulement jusqu'au $\mathrm{X}^{\mathrm{e}}$-, les visiteurs musulmans de l'Afrique orientale n'ont guère pénétré au sud du Sénégal et du Niger, au sud d'Assouan, dans la vallée du Nil; s'agissant des côtes de l'Afrique orientale, l'équateur n'est vraisemblablement régulièrement dépassé qu'à partir du IX ${ }^{\mathrm{e}}$ siècle« (DEVISSE, Islam et ethnies [voir n. 6], p. 107).

13 Vincent MonTEIL, Al-Bakri (Cordoue 1068), routier de l'Afrique blanche et noire du NordOuest, Dakar 1968.

14 Al Maghili fit, au début du XVI ${ }^{\mathrm{e}}$ siècle, un inventaire dans le pays des Noirs au profit de l'empereur Songhay et pour le salut de la Umma, pour désigner clairement qui est musulman et qui ne l'est pas. Lire Jean CUOQ, Recueil des sources arabes concernant l'Afrique occidentale du VIII ${ }^{\mathrm{e}}$ au XVI ${ }^{\mathrm{e}}$ siècle, Paris 1975, p. 412-423.

15 »Quant aux Toucouleurs, ce mot perpétue l'ancien >Tekrour〈 (takrour) >ambulantく des auteurs arabes au moyen Âge, depuis Al Bakri et le $\mathrm{XI}^{\mathrm{e}}$ siècle« (Vincent MONTEIL, L'Islam noir, Paris ${ }^{2} 1971$, p. 84-85).

16 Ca Da Mosto, Relation de voyages à la côte occidentale d'Afrique: 1455-1457, publiée par Christian SCHEFFER, Paris 1895, p. 33.

17 Yoro FALL, L'Afrique à la naissance de la cartographie moderne ( $\mathrm{XIV}^{\mathrm{e}}-\mathrm{XV}^{\mathrm{e}}$ siècles), Paris 1988, p. 122. 
trionale, les Européens vont désigner les marabouts musulmans sédentaires de la vallée par le terme »tooroodo«.

\section{LES EUROPÉENS AU CONTACT DE L'INTÉRIEUR DE L'AFRIQUE AU XIX ${ }^{\mathrm{e}}$ SIÈCLE ET LEUR PERCEPTION DE L'ALTÉRITÉ}

Le mandat du gouverneur de la colonie du Sénégal Julien Schmaltz (1816-1820) est marqué par une volonté de rattraper le temps perdu après l'intermède des Anglais (de 1759 à 1779 et de 1807 à 1816)18. Mais le gouverneur n'avait pas les moyens militaires de »civiliser les peuplades riveraines du fleuve Sénégal«19. Il doutait d'ailleurs des capacités des Noirs: »Quoique parfois très subtile dans les détails, leur politique n'embrasse pas d'ensemble. À cause de leur défiance, de l'inconsistance de leur caractère « 20 .

$\mathrm{Au}$ début du XIX $\mathrm{X}^{\mathrm{e}}$ siècle, Gaspard Théodore Mollien (1796-1872) s'intéressa à l'exploration de la Sénégambie. Mollien, fils d'un procureur au Parlement français, commis de la marine quand il gagna le Sénégal pour un premier séjour en 1816, un an après la restitution de la colonie. En 1818, il entreprit volontairement un voyage avec comme ambition la découverte des sources du Sénégal, de la Gambie et du Niger au Fuuta Jalon. L'expédition géographique dura un an et révéla les sources du Rio Grande, de la Gambie et de la Falémé. Il visita le Kajoor, le Fuuta Tooro, le Joloof etc... 11 avait un guide qui s'exprimait en arabe, en pulaar et en wolof, lui-même s'exprimant presque parfaitement en wolof. Son récit de voyage, avec carte et vue à l'appui, fut publié pour la première fois en 1820, un an après la fin de son exploration au Fuuta Jalon. La deuxième édition datant de 1822 est de l'avis même de l'auteur la plus correcte.

Mollien insiste sur ce qui différencie les populations:

En effet, tous les États mahométans que j'ai l'occasion de visiter sur la côte d'Afrique sont fédératifs, tandis que les peuples païens gémissent sous la tyrannie la plus atroce. Ainsi les Peules et les Mandingues jouissent d'un gouvernement doux, les Iolofs sont continuellement exposés aux caprices farouches de leurs souverains ${ }^{21}$.

Un autre auteur continua sur cette lancée et utilisa dans ses écrits le terme »Toucouleur«. Il s'agit de David Boilat; né le 20 avril 1814 à Saint-Louis du Sénégal, celui-ci était un missionnaire catholique métis franco-africain. Il fut confié à la mère Javouhey par son père. Elle l'envoya en 1827 en France pour ses études religieuses. En 1841, il reçut son ordination en France et en 1843 il retourna à Saint-Louis, sa ville natale, où il

18 Mamadou Diouf, Le Kajoor au XX ${ }^{\mathrm{e}}$ siècle. Pouvoir ceddo et conquête coloniale, Paris 1990.

19 Archives nationales du Sénégal (ANS), 2B24, Gouverneur Ollivier au ministre des Colonies, Saint-Louis le 4 avril 1846, dépêche n 148.

20 ANS, 2B5, Gouverneur Schmaltz au secrétaire d'État de la Marine et des Colonies, SaintLouis, le 27 mars 1820, dépêche n 82.

21 Gaspard Théodore Mollien, L'Afrique occidentale en 1818, London 1820, p. 65. 
fonda une école secondaire22. Deux ans plus tard, il se réinstalla sur l'île de Gorée afin d'étudier »l'histoire et les sociétés de l'intérieur « ${ }^{23}$. Il a été victime de ses >frères〈 de Ploermel et ceux du Saint-Esprit. Il y a dans ce conflit des relents racistes.

Son livre, intitulé »Les Esquisses sénégalaises. Physionomie du pays, peuplades, commerce, religion, passé et avenir, récits et légendes«, parut en 1853, dix ans après son retour au Sénégal et est considéré comme la première production littéraire d'un Sénégalais en français. Les douze chapitres du premier volume se répartissent suivant les différentes régions du Sénégal et leurs populations.

La confusion dans les tentatives de présentation des peuples fut entretenue par les visiteurs européens au XIX ${ }^{\mathrm{e}}$ siècle, entrés en contact avec les Fuutankoobe, qui choisirent de prime abord comme élément d'altérité la couleur de la peau! De ce point de vue, d'une part »le vrai type et sans mélanges« se rencontre parmi les Peuls »couleurs bronze, rouge « qui »sont des hommes sans nations, sans chefs«, d'autre part les croisements avec les Noirs engendrent les Toucouleures, qui sont des »fanatiques rigides observateurs du Koran «24. L'appellation »Tukulër« (Toucouleur), un élément d'identité à base religieuse, était promise à un bel avenir.

Deux facteurs influencèrent les études sur la pensée raciale. Il s'agit d'une part de la naissance et du développement de l'anthropologie physique française. En effet, en France, de nombreuses sociétés savantes furent créées à partir de la deuxième décennie du XIX ${ }^{\mathrm{e}}$ siècle dans les domaines de la géographie et de l'anthropologie: la Société de géographie de Paris fut fondée à Paris en 1821, éditant dès 1822 sa revue, le »Bulletin de la Société de géographie«. La Société ethnologique de Paris fut fondée en 1839; la Société d'ethnographie française en 1847; en 1859, naquit la Société d'anthropologie de Paris avec sa publication, le »Bulletin de la Société d'anthropologie de Paris«. En dehors des sociétés savantes, d'autres publications, comme les »Nouvelles Annales de voyages« (1819-1865), un recueil de traductions de voyages inédits, ou la »Revue coloniale« (1843-1858) virent le jour. Un intérêt supplémentaire fut donné à l'Afrique et à sa population.

\section{LES MANDATS DE FAIDHERBE COMME GOUVERNEUR DE LA COLONIE (1854-1861, 1863-1865) ET LA DOUBLE STIGMATISATION DU NOIR}

Nommé gouverneur en décembre 1854, Faidherbe entreprit plusieurs travaux ethnographiques afin de mieux connaître les populations. Il créa dès sa nomination deux

22 Certainement la première école secondaire de Saint-Louis. Jusqu'en 1903, les congréganistes contrôlaient l'école dans la colonie. Lire Mamoudou Sy, École française contre daara (école coranique) au XIX ${ }^{\mathrm{e}}$ siècle. La volonté de civiliser les peuples de la Sénégambie, publié par Institute for the Study of Islamic Thought in Africa (ISITA), Northwestern University, Evanston, Institut islamique de Dakar, Actes de l'atelier: L'enseignement islamique au Sénégal: Histoire, transformations et actualités, Dakar, 8-10 août 2007.

23 Abbé BolLat, Esquisses sénégalaises, Paris 1853, p. 34.

24 Ibid., p. 384-385. 
périodiques: »Annuaire du Sénégal et dépendances« et »Moniteur du Sénégal et dépendances« destinés à la publication des actes officiels, mais aussi des essais historiques, anthropologiques, économiques et politiques.

Fin novembre $1855^{25}$, à l'occasion d'une réception offerte en son honneur par les notables de la ville de Saint-Louis pour le féliciter des grandes réalisations opérées en moins d'un an de magistère, le gouverneur Faidherbe prononça un discours, qui resta la feuille de route pour lui et ses successeurs à la tête de la colonie. Faidherbe expliquait que c'était le caractère instable de la présence française qui avait causé une fluctuation extrême des relations politiques avec les peuplades riveraines de la vallée du Sénégal ${ }^{26}$. Ainsi, déclairait Faidherbe, »le nom français, partout dédaigné était devenu synonyme de tributaire, et les Maures qui habitent la rive droite du fleuve Sénégal avaient profité de cette faiblesse apparente pour dominer et dépeupler la rive des Noirs «, c'est-à-dire la rive gauche27. À son avis, il était injuste que la France, »cette vieille et noble nation qui marche depuis des siècles à la tête de la civilisation du monde, paye un tribut religieux à des peuplades sauvages qui ont appris depuis quelques années le Koran? «28 Il allait se charger de remettre ces populations blanches (Maures) ou noires (Wolof, Fulbe, Soninke) à leur place.

À deux reprises, dans son discours, le gouverneur Faidherbe prononce le terme "peuplade« et, la deuxième fois, il met les pieds dans le plat en y adjoignant le terme »sauvage«. Alors il fallait les faire rentrer dans le monde civilisé avec un préalable, leur annexion à la colonie. Il entreprit la conquête militaire de la Sénégambie et ajouta d'abord en 1855 le royaume du Waalo à la colonie. Faidherbe fit aussi de l'impérialisme linguistique et culturel une priorité.

L'école française était un moyen important, efficace pour extirper ces peuplades de l'état sauvage. En 1856, il créa l'École des otages ${ }^{29}$ pour enrôler les fils de chefs traditionnels africains et catalyser l'expansion du rayonnement de la civilisation française dans ce monde de ténèbres. La même année, il commit dans la »Revue coloniale« un article intitulé »Populations noires des bassins du Sénégal et du Haut-Niger« 30 . Il voulait »présenter le tableau actuel d'une partie des contrées de l'Afrique occidentale«. Il insistait sur la distinction des races du Soudan occidental d'une manière générale.

En 1857, Faidherbe prit un arrêté qui contrôlait et réglementait les daaras (écoles coraniques), qui étaient la base de l'éducation islamique ${ }^{31}$. Pourquoi? Parce que, selon l'administration coloniale, ces écoles coraniques transformaient vicieusement la mentalité des jeunes Africains et constituaient un frein au rayonnement de la civilisation

25 Exactement le 11 novembre 1855, lors d'un banquet offert par la bourgeoisie locale.

26 Moniteur du Sénégal et dépendances, 1855-1859.

27 Il faut noter que c'est Faidherbe qui a théorisé et imposé la transformation du fleuve Sénégal en frontière entre la Négritie et la Mauritanie. Ce qu'il n'a jamais été.

28 Moniteur du Sénégal et dépendances, 1855-1859.

29 Qui devint en 1892, après une période de léthargie, l'École des fils de chefs et des interprètes.

30 Louis FAIDHERBE, Populations noires des bassins du Sénégal et du Haut-Niger, dans: Revue coloniale, $2^{\mathrm{e}}$ série, 16 (1856), p. 328-341.

31 Pour plus de détails, lire SY, École française (voir n. 22). 
française. La même année, il mit sur pied le bataillon des tirailleurs sénégalais pour éduquer cette classe d'âge qui avait échappé à l'école.

En 1858, le général Faidherbe prit une décision consistant à changer l'appellation d'un service de la colonie: »la direction des Affaires extérieures«, chargée des relations avec les États sénégambiens, en »direction des Affaires indigènes«. Le terme »indigène« montre le caractère inférieur que le gouverneur donne à ces peuples, légitimant ainsi l'entreprise coloniale. En 1859, il publia un second livre de 99 pages ayant pour titre: »Notice sur la colonie du Sénégal et sur les pays qui sont en relation avec elle«.

Les portes de l'école et de l'armée que Faidherbe ouvrit aux Noirs leur assuraient des perspectives de carrière minable. Le général Faidherbe fut, au total, l'auteur d'une quinzaine d'ouvrages relatifs à l'anthropologie, à l'histoire, aux parlers pulaar, wolof, soninke, berbère, arabe, hassania et sereer. Les travaux sur les peuples africains comportaient souvent un élément d'appréciation sur leur aptitude au travail, leur docilité et les produits qu'ils pouvaient fournir à la colonie. L'essentiel était de les faire travailler au maximum; ce qui était difficile, car d'après le gouverneur Brière de l'Isle: »Les Noirs sont difficiles à faire sortir de leur apathie et de leur paresse « ${ }^{32}$.

Les administrateurs coloniaux, préoccupés au $\mathrm{XIX}^{\mathrm{e}}$ siècle à faire subir aux »peuplades« de la Sénégambie la domination française, étaient convaincus cependant que celles-ci traînaient au moins deux tares: soit elles étaient noires et musulmanes, soit elles étaient noires et païennes!

Une guerre sainte fut menée par El Hajji Umar Taal (né vers 1796 et mort en 1864) qui symbolisa le tooroodo typique aux yeux des Français: violent, fanatique et guerrier. Selon le capitaine Flize de l'état-major de la colonie du Sénégal:

Les gens du Dimar ont été par le passé la terreur des traitants français. Musulmans fanatiques et orgueilleux, ils doivent à la pratique des lois du Koran des garanties dont manquent leurs voisins du Walo et du Kajor, soumis aux seuls caprices des chefs grossiers et despotes; mais ils ont aussi poussé à l'excès les vices qu'engendre l'islamisme ${ }^{33}$.

Analysant l'action d'El Hajji Umar Taal, le général Faidherbe, un ancien de l'Algérie qui influença beaucoup l'administration locale dans la théorie de l'encerclement de la colonie du Sénégal par des forces musulmanes hostiles, disait:

El Hajji Umar, toorodo des environs de Podor, prêcha une guerre sainte et après avoir été repoussé par nous de la partie navigable du Sénégal, entreprit la fondation d'un vaste empire peul musulman occidental sur le haut Niger et le haut Sénégal pour faire le pendant de l'empire peul oriental fondé par Othman Dan Fodio ${ }^{34}$.

Faidherbe, se demandant si la conversion à l'islam était un progrès pour les Noirs, précisera: »quoique ses imperfections deviennent si flagrantes au contact d'une société

2 ANS, 2B46, gouverneur Brière de l'Isle au ministre, Saint-Louis, 7 novembre 1878.

33 Moniteur du Sénégal et dépendances, n 110, 4 mai 1858.

34 Moniteur de l'Algérie, Alger $1^{\text {er }}$ septembre 1866, général Faidherbe. 
plus éclairée «35. Il faisait allusion aux Arabes, aux Berbères et aux Turcs, qui dans son entendement sont plus éveillés que les Noirs. Pour donner une facette du caractère infantile du Sénégambien, il donne un exemple:

On peut citer 1000 faits atroces à l'appui de ce que nous avançons; contentons nous de celui-ci qui n'est que comique. Daou Demba, roi du Cayor (royaume Wolof), en 1640, défendit à ses sujets de saler les aliments parce qu'il n'était pas convenable que de simples sujets se servissent d'un condiment dont le roi faisait usage ${ }^{36}$.

Faidherbe initia en 1858 une campagne pour susciter la haine entre les différents peuples sénégambiens, lorsque El Hajji Umar revint en Sénégambie septentrionale. Faidherbe et son équipe du bureau politique ${ }^{37}$ adressèrent une série de correspondances anti-umariennes aux rois et notables (wolof) du Kajoor ${ }^{38}$, aux habitants du Njambur ${ }^{39}$, aux tribus (mauresques) de l'est du Sahara ${ }^{40}$, aux chefs des pays wolofs, au Buur ba Jolof ${ }^{41}$, etc. Le contenu est le même: »El Hadji a détruit tout le pays de l'Est. Comme il n'y a plus de villages, de mil, de troupeaux et de pillages à faire que dans ce pays où il vient, ce sont vos biens qu'il veut. Si vous êtes assez sots pour vouloir vous laisser faire... «42. Pour mieux ferrer ses correspondants, le discours faisait allusion à l'étiquetage ethnique:

Les Toucouleurs du Fouta qui n'ont ni travaillé ni fait du commerce depuis trois ans sont dans la plus grande misère. Ils ont dévoré tous les pays de l'Est, tiédos [païens] ou marabouts. [...] les Toucouleurs voudraient venir dévorer les pays ouoloffs qui sont riches parce qu'ils travaillent et font du commerce. Mais les Ouoloffs ne les laisseront pas faire et nous irons au secours des Ouoloffs... ${ }^{43}$

Et après Faidherbe? La même pensée à l'égard du Noir continue. La présentation des toorobbe d'une manière particulière et des Toucouleurs d'une manière générale comme un peuple fanatique, orgueilleux par Flize, puis Faidherbe est confirmée par le gouverneur Valière:

35 Ibid.

36 Ibid.

37 Flize, Bou El Mogdad, etc.

38 ANS, 3B91, correspondance de Flize à Damel, en date du 3 juillet 1858, pièce 8; correspondance de Faidherbe à Damel, en date du 3 février 1860, pièces 64/65, et du 15 mars 1860, pièce 57.

39 ANS, 3B91, correspondance de Faidherbe aux gens du Ndiambour, en date du $1^{\text {er }}$ septembre 1858, pièce 9, en date du 17 septembre 1858, pièce 56 versos, correspondance de Faidherbe aux Maures Sidi Makhmoud; ANS, 3B92, correspondance de Faidherbe à Sidi Mouhamed en date d'août 1858 .

40 ANS, 3B92, correspondance de Faidherbe aux tribus de l'Est, 1859, lettre n 1.

41 ANS, 3B92, correspondance de Faidherbe, en date du $1^{\text {er }}$ septembre 1858, pièce 22 verso.

42 ANS, 3B91, correspondance de Faidherbe aux gens du Ndiambour, Saint-Louis, en date du $1^{\text {er }}$ septembre 1858 , pièce 9 .

43 ANS, 3B92, correspondance de Faidherbe aux chefs et habitants ouoloffs, Saint-Louis, en date du $1^{\text {er }}$ septembre 1858 , pièce 22 verso. 
Ces interminables guerres intestines qui ensanglantent constamment le Fouta dérivent de la situation politique de ce pays, du caractère et des usages de ses habitants: d'ailleurs si les Toucouleurs n'étaient pas tous à leurs luttes intestines, ils porteraient la guerre chez leurs voisins, tant l'esprit de cette population est turbulent et son humeur guerrière ${ }^{44}$.

Un autre groupe ethnique est aussi évalué. Selon le gouverneur de la colonie du Sénégal, Valière, les Mandingues sont paresseux, voleurs et très turbulents ${ }^{45}$. Jauréguiberry, gouverneur de la colonie quelques années plus tôt, était sceptique quant aux capacités des Noirs à s'adonner à des activités économiques. En 1862, il proposa à ses supérieurs hiérarchiques l'installation sur la bande côtière s'étendant de Lompoul au Cap-Vert c'est-à-dire de Louga à Dakar dans la zone des Niayes - de quelque 20000 émigrés chinois et indiens, qui deviendraient propriétaires terriens au bout de trois ans de séjour! Il suggéra aussi que soient dirigés vers ces contrées des esclaves fugitifs de la Virginie qui devaient aller à Haïtite. Il insista sur la violence qui perdurait en Afrique, mais s'abstint d'en donner toutes les causes.

Ces guerres ensanglantent le continent africain, dont le trafic des esclaves ou des mœurs barbares ont été le mobile. Autour de nous, dans la Sénégambie proprement dite, cet abandon des cultures peut être attribué aux vices des coutumes locales, qui ne garantissent pas l'agriculture contre les spoliations des chefs ou des guerriers ${ }^{47}$.

À la fin des années 1870 , le gouverneur Brière de l'Isle, qui resta à la tête de la colonie de 1876 à 1881, entretint, au dehors de la Sénégambie, une politique de confrontation entre les Toucouleurs et les Bambara, les premiers étant considérés comme des envahisseurs des seconds. Il était encouragé par la propagande coloniale des maisons de commerce bordelaises et marseillaises, par l'action des républicains et enfin par le ministre de la Marine et des Colonies, Jauréguiberry.

Dans le cadre du projet français d'expansion coloniale vers l'Est, beaucoup d'anthropologues, de voyageurs, de militaires vont sillonner le Soudan occidental avec comme principal but d'apporter la civilisation dans ce continent considéré comme étant habité par des sauvages.

C'est le cas des missions Mage et Quintin, Gallieni, Soleillet, etc. La mission d'Eugène Mage vers le haut Niger entre 1863 et 1866 est illustrative de ce dessein. Analysant en septembre 1866 depuis Alger, ses enjeux, Faidherbe, fidèle à sa feuille de route dressée en 1855 lors du banquet offert en son honneur, disait:

Le vieux monde africain, régénéré par la demi-civilisation musulmane, galvanisé par le fanatisme, pressent que c'est par cette brèche de la vallée du Sénégal que la race européenne et son cortège

44 ANS, 3B40, gouverneur Valière au ministre des Colonies, Saint-Louis, mars 1873.

45 Ibid.

46 ANS, 2B33, gouverneur Jauréguiberry au ministre des Colonies, Saint-Louis, 7 mars 1862, pièce 126, Rapport sur la culture des terrains au Sénégal. Demande d'émigrants chinois et indiens.

47 Ibid. 
d'idées et d'institutions pénétrera avant peu jusqu'au cœur de ce continent arriéré, et, par l'instinct de conservation naturel à toute chose, il cherche à se défendre de cette invasion ${ }^{48}$.

D'autres individus vont emboîter le pas à Mage et Quintin. Joseph Simon Gallieni (1849-1916) a des origines italiennes, mais a grandi avec les valeurs républicaines de la Révolution française. C'est un ancien de la Flèche et de Saint-Cyr. Vétéran de la guerre franco-allemande, il prit contact avec l'Afrique à la Réunion, comme lieutenant de l'infanterie de marine, avant de servir au Sénégal en 1876. Il fut brièvement commandant de poste puis servit au bureau politique, avant d'entreprendre deux voyages d'exploration sous le commandement du gouverneur du Sénégal Brière de l'Isle.

Gallieni fut chargé en 1879 d'étudier le tracé de la future voie ferrée MédineBafoulabé. Il signa des traités avec des chefs de la haute vallée du Sénégal et créa le poste de Bafoulabé. Son second voyage se déroula de 1880 à 1881 en compagnie de deux officiers géographes-topographes et de deux médecins, Tautain ${ }^{49}$ et Bayol, qui rédigèrent leurs mémoires de voyage.

Les objectifs de cette mission sont contradictoires en ce sens que, à coté des ambitions politiques et commerciales, le capitaine Gallieni comptait œuvrer pour »exploiter les ferments de discorde entre la race des conquérants toucouleurs et leurs tributaires «. Mais Gallieni, sur la route de la capitale d'Ahmadou Taal, fut arrêté à Nango, près de Ségou, où les membres de l'expédition séjournèrent pendant dix mois. Il eut le temps de connaître les peuples africains. En avril 1880 fut signé le traité de Kita, et en mars 1881 Ahmadou ratifia le traité de Nango. Gallieni reçut la médaille d'or de la Société de géographie.

En 1883, dans la revue »Tour du monde«, le commandant Gallieni publia ses mémoires relativement à cette mission. À propos du traité de Kita, reproduit intégralement, il est stipulé à l'article premier: »Les chefs, notables et habitants du pays de Kita déclarent qu'ils vivent indépendants de toute puissance étrangère et qu'ils usent de cette indépendance pour placer de leur plein gré, eux, leur pays et les populations qu'ils administrent sous le protectorat exclusif de la France ${ }^{50}$. Gallieni réussissait dans son objectif de semer la discorde entre populations africaines. Il dirigea par la suite plusieurs campagnes militaires, notamment au Niger, sous le grade de capitaine. À son tour, il commandita des voyages d'exploration exécutés vers le pays Malinke par Péroz, en pays peul jalonke par Plat et en pays peul masina par Caron. Il devint ensuite gouverneur du Soudan en 1886, du Tonkin en 1893 puis de Madagascar en 1896. Il fut fait maréchal à titre posthume en 1921.

Louis Joseph-Marie Quintin, chirurgien de la marine affecté au Sénégal en 1860, accompagna aussi Gallieni et publia en 1881 un article dans le »Bulletin de la Société de

48 Extrait du Moniteur de l'Algérie, Général Faidherbe, Alger, $1^{\text {er }}$ septembre 1866.

49 Docteur Louis-Frédéric TAUTAIN, Études critiques sur l'ethnologie et l'ethnographie des peuples du bassin du Sénégal, publiées sous les auspices du ministère de l'Instruction publique et des Beaux-Arts par le docteur Hamy, Paris 1885, p. 1-45.

50 Joseph GALLIENI, Exploration du haut Niger, dans: Le Tour du monde $\left(1883,1^{\text {er }}\right.$ semestre), p. 116. 
géographie« intitulé »Étude ethnographique sur les pays entre le Sénégal et le Niger«. En annexe est jointe une carte des populations de la Sénégambie et du haut Niger.

Paul Soleillet (1842-1885) séjourna dix ans en Algérie, puis arriva au Sénégal en 1878 dans le but d'ouvrir l'Afrique occidentale au commerce et à l'influence civilisatrice de la France. Son voyage fut financé par le ministre de l'Instruction publique. Il quitta Saint-Louis en avril 1878 et resta quatre mois à la cour d'Ahmadou Taal à Ségou - comme avant lui Mage et Quintin - puis repartit par Nioro en mars 1879. Le récit de son voyage parut en 1886, sept ans après son retour et un an après sa mort, sous le titre »Voyage à Ségou (1878-1879)«; il fut rédigé d'après ses notes et ses journaux de voyage par Gabriel Gravier.

Cette entreprise de confrontation des peuples ouest africains atteint son paroxysme en avril 1890 lorsque la prise de Ségou par Archinard entraîna la chute de l'État toucouleur et l'expulsion de milliers de Fuutankoobe vers la vallée du Sénégal, dont la majorité mourut sur le chemin du retour.

\section{CONCLUSION}

Les efforts déployés pour se rattacher à des ancêtres extérieurs à l'Afrique servirent à justifier la possession et la conservation d'un pouvoir et d'un savoir inspiré de la pensée islamique par une frange de la population locale, les Toroodo, appartenant aux Pulaar de la vallée du Sénégal. Par ailleurs, pour légitimer la colonisation européenne, les Français devaient prouver avec le recours à un corpus scientifique le caractère souscivilisé de tous les peuples de la Sénégambie en particulier et de l'Afrique en général. Cette contradiction dans les représentations n'a pas varié durant tout le $\mathrm{XIX}^{\mathrm{e}}$ siècle. Les idées de domination seront reprises par Jules Ferry à la fin du XIX ${ }^{\mathrm{e}}$ siècle puis par Albert Sarraut dans les années 1920. La pensée raciale du colon vis-à-vis du colonisé africain a une constante: le mépris de l'homme noir et la certitude qu'il est un être inférieur que l'on doit dominer. Ce qu'il faut regretter et dénoncer, c'est qu'on assiste en Afrique, aux $\mathrm{XX}^{\mathrm{e}}$ et $\mathrm{XXI}^{\mathrm{e}}$ siècles, dans certains pays, à une politique qui est à l'opposé de celle des Toroodo et qui consiste dans une revendication à l'autochtonie. Cela entraîne malheureusement la chasse à »l'étranger« avec toutes ses conséquences néfastes. 